\title{
Escuelas de tiempo completo y logro educativo: experiencias de las escuelas PETC en San Luis Potosí periodo 2013-2017
}

\section{Full-time schools and educational achievement: experiences of PETC schools in San Luis Potosí from 2013 to 2017}

\author{
Armando René Espinosa Hernández ${ }^{1}$ \\ Olga Leticia Álvarez Cooper²
}

\begin{abstract}
Resumen
Este trabajo revisa el funcionamiento de los componentes del Programa de Escuelas de Tiempo Completo en 39 instituciones participantes, vinculándolo con los resultados del logro educativo. Metodológicamente se realizó un trabajo cualitativo con entrevistas, observación de la práctica docente, y revisión documental. Se sustenta en los postulados de la Gestión Escolar de Pozner (1997). Uno de los principales hallazgos el relativo efecto que han tenido el PETC en los resultados educativos.
\end{abstract}

\section{Palabras clave}

Logro educativo, escuelas, jornada escolar completa, programa.

\begin{abstract}
This paper reviews Full-Time School Program effect in 39 participating institutions, linking it with educational achievement results. Methodologically, a qualitative work was carried out with interviews,

1 Armando René Espinosa Hernández. Investigador educativo en el Sistema Educativo Estatal Regular, San Luis Potosí, México. Sus líneas de investigación son: Historia de la Educación y del Sindicalismo Magisterial, así como temas de las Reformas Educativas en México. Correo electrónico: dieseer@gmail.com ID: http://orcid.org/0000-0003-2352-3245
\end{abstract}

2 Olga Leticia Álvarez Cooper. Programa Estudios Latinoamericanos en Territorio, Sociedad y Cultura de la Universidad Autónoma de San Luis Potosí, México. Es licenciada en comercio exterior y magíster en educación por la Universidad del Centro de México. Correo electrónico: llqper@alumnos.uaslp.edu.mx.

ID: http://orcid.org/0000-0003-1909-7431 
RECIE. Revista Electrónica Científica de Investigación Educativa Vol. 4, núm. 2, enero-diciembre 2019, pp. 943-954.

observation of the teaching practice, and documentary review. It is based on Pozner's (1997) School Management postulates. One of the main findings is the relative effect that PETC has had on educational outcomes.

\section{Keywords}

Educational achievement, full-time school, program.

\section{Introducción}

En el año escolar 2015-2016 el Departamento de Investigación Educativa y el Técnico Pedagógico del Sistema Educativo Estatal Regular (SEER) fueron designados para hacer una revisión de los avances del Programa de Escuelas de Tiempo Completo (PETC), en 37 instituciones de educación primaria en el estado de San Luis Potosí.

La información que detonó la preocupación en las autoridades educativas fueron los resultados obtenidos de las escuelas participantes en el PETC en la evaluación de la Olimpiada del Conocimiento Infantil (OCI) eran desalentadores, se observaba que los porcentajes obtenidos en la mayoría de las escuelas iban a la baja, en otras más no se percibía ninguna mejora y pocas eran las que estaban avanzando.

La ampliación de la jornada escolar es una política utilizada por diversos sistemas escolares alrededor del mundo, pues parece que entre más permanezcan los alumnos en la escuela la calidad de los aprendizajes mejor, no es un hecho en automático, el tiempo adicional tiene que ser utilizado en actividades propias de la gestión pedagógica, de los programas de estudio, y aspectos en donde al alumnado manifiesta rezago, en términos generales se tendrían que atender las cuatro dimensiones de la gestión escolar (Pozner, 1997).

Emilio Tenti (2010) a propósito de la implementación del programa PECT en México, encontró una diversidad de formas de organizar la jornada escolar; que están relacionadas con las responsabilidades en cada de uno de los niveles de gobierno; tradiciones educativas; intereses gremiales y de la ciudadanía; la posibilidad de acceder a recursos financieros; modificación de las condiciones laborales del magisterio y las adaptaciones curriculares.

A pesar de que todos los sistemas educativos, parten de que a más permanencia en la escuela mejorarán los aprendizajes, Tenti es contundente al afirmar; que cuando menos las reformas educativas comprendidas entre 1985-2000, no se guiaron por esa premisa (2010, p. 13-14). Es hasta tiempos recientes que ha surgido el interés por el tema, así, tanto países europeos como latinoamericanos han "identificado el aumento del tiempo 
escolar como un factor central a la hora de mejorar la calidad educativa y la equidad" (Tenti, 2010, p. 23).

Diversos estudios de corte etnográfico y documental han encontrado que las escuelas de jornada ampliada refuerzan la permanencia de los alumnos en el sistema escolar, disminuyendo el ausentismo y el rezago, sobre todos aquellas que cuentan con servicio de comedor, que tiene efectos positivos sobre la equidad. También han revelado limitaciones como; la usencia de liderazgo pedagógico o educativo de los directores de las escuelas, desconocimiento o aplicación inadecuada del componente pedagógico; asimismo de una creciente responsabilidad en el trabajo administrativo. Además, se han centrado generalmente en casos de éxito (SEP, 2009; López 2012; Veleda 2013; Gómez, 2015; Martinic, 2015) pocos se sitúan en una posición crítica, que cuestione el modelo o programa como lo hace Fernando Ramírez Llorens (2014) que trata la extensión de la jornada escolar en Uruguay.

Para el caso específico que nos ocupa se tomaron en cuenta las siguientes interrogantes: ¿Qué tanto conocen los docentes el enfoque pedagógico del PETC? ¿Cómo aplican los materiales educativos proporcionados por el programa? ¿Cómo incluyen las líneas de trabajo educativo en la planeación didáctica los docentes? ¿Cuáles fueron los resultados del logro educativo de las escuelas participantes en el PETC? ¿Cómo utilizan el tiempo de la jornada escolar ampliada? Todas relacionadas con el componente pedagógico del PETC.

La investigación es guiada por el supuesto de trabajo que afirma; Los resultados del logro educativo en las escuelas participantes del PETC, están condicionados por la forma de selección de las escuelas, el conocimiento, dominio y aplicación del componente pedagógico del programa por parte del colectivo docente.

Objetivo fue: Revisar el funcionamiento de los componentes del PETC, con énfasis en el pedagógico, en las escuelas participantes, vinculándolo con los resultados del logro educativo, con la finalidad de formular recomendaciones para mejorar su implementación.

El PETC es un programa contemplado en el Plan Nacional de Desarrollo y en el Programa Sectorial de Educación, con objetivos y metas, como; asegurar mayor cobertura, inclusión y equidad educativa entre los grupos poblacionales; ofrecer más y mejor tiempo a los alumnos, optimizar el ingreso, retención y aprovechamiento escolar, además de proporcionar atención a la población más pobre (GR. s.f.; PSE, 2013). En forma resumida los componentes más relevantes son; ampliación de la jornada escolar, las

Escuelas de tiempo completo y logro educativo: experiencias de las escuelas... 
RECIE. Revista Electrónica Científica de Investigación Educativa Vol. 4, núm. 2, enero-diciembre 2019, pp. 943-954.

líneas de trabajo educativo y sus materiales correspondientes, el servicio de alimentación, la remuneración y la administración del programa (SEP, s. f.)

El objetivo general es: "Mejorar la calidad de los aprendizajes de las niñas y los niños en un marco de diversidad y equidad, propiciando el desarrollo de las competencias para la vida y el avance gradual en el logro del Perfil de Egreso de la Educación Básica, a través de la ampliación y uso eficiente del tiempo, el fortalecimiento de los procesos de gestión escolar y las prácticas de enseñanza, así como la incorporación de nuevos materiales educativos" (SEP, s. f., p. 11).

La aplicación del programa implica que las escuelas amplíen sus horarios hasta completar de 6 a 8 horas diarias, por lo tanto, un horario sería de 8:00 a $14.30 \mathrm{hrs}$ y el segundo se extendería hasta las 16: $00 \mathrm{hrs}$., los alumnos cuentan con 30 minutos de receso para jugar al aire libre y la ingesta de un refrigerio, si se cuenta con el servicio de comedor la alimentación se proporcionara después terminar la jornada escolar. El tiempo adicional se debe de ocupar en fortalecer los aprendizajes de los alumnos a través de profundizar en el tratamiento de los contenidos de las asignaturas y en desarrollar las Líneas de Trabajo Educativo, que son explicitadas en los ficheros de actividades didácticas denominados; Jugar con números y algo más, Leer y escribir, Leer y escribir en lengua indígena, Aprender a convivir, Vivir saludablemente, Expresar y crear con arte, Aprender con TIC. Además de lo anterior hay un conjunto de responsabilidades que concierne a los directores, docentes, alumnos, padres de familia, Consejo Técnico Escolar, Supervisión y Consejo Escolar de Participación Social. (SEP, s. f., p. 25-38).

El contenido general del programa se relaciona con las cuatro dimensiones de la gestión escolar propuestas por Pozner (1997), pues para ser una escuela seleccionada, se requiere de elaborar un proyecto de trabajo por el Consejo Técnico Escolar al que se le denomina Ruta de Mejora, este toma en el progreso educativo, contextualiza los problemas de aprendizaje, establece acciones puntuales para abatir el rezago, así como atender a los alumnos en desventaja y vulnerabilidad, incorpora la Líneas de Trabajo Educativo. Se le encomienda al Consejo de Participación Social involucrarse en tareas como; la preparación de los alimentos de acuerdo, generar apoyos por los padres de familia, exalumnos, líderes de la comunidad, además de operar actividades de índole escolar y extraescolar que fortalecen el PETC. Los directores en conjunto con la supervisión son responsables de administrar y rendir los informes necesarios de los recursos asignados a la escuela, y también responden por la organización y el funcionamiento de la 
escuela en todos sus aspectos. Como se puede observar en la descripción de las responsabilidades de los integrantes de la comunidad educativa están presentes las dimensiones; pedagógica-curricular, comunitaria, administrativa financiera y la organizacional-operativa.

La información obtenida para la etapa de diagnóstico de basó en las siguientes acciones; entrevistas a profundidad con los funcionaros responsables del programa en un formato abierto; un trabajo documental para revisar las reglas de operación publicadas en el Diario Oficial de la Federación, los Lineamientos para la Operación y Organización de las Escuelas de Tiempo Completo en Educación Primaria, las líneas de trabajo educativo con su fichero de actividades didácticas, ubicación geográfica de las escuelas y su correspondencia con el nivel de marginación, resultados de la OCI, además de sostener reuniones de información con los supervisores y directores, también se consideró un grupo de análisis, reflexión y propuesta, el cual tuvo como tarea la revisión de toda la información obtenida, con la finalidad de armar un documento de diagnóstico ejecutivo que fuera muy entendible y práctico para la toma de decisiones posteriores al diagnóstico.

A partir del ciclo escolar 2013-2014 se inició un proceso de incorporación de escuelas al PETC, en ese año solamente se contaba con dos, pero para el ciclo escolar 2016-2017 se incrementaron a 39, en general están ubicadas en localidades de muy alto y alto nivel de marginación (23); siete están en índice medio, cinco en bajo, una en muy bajo y tenemos tres identificadas en Zonas Urbanas de Atención Prioritaria (ZAP) (Ley General de Desarrollo Social, 2013, p. 7).

El proceso para que las escuelas se inscriban en PETC ha seguido dos vertientes; una la administración educativa federal identifica que la población que habita la localidad cumple con cualquiera de los siguientes requisitos; se ofrezcan los servicios de educación primaria o telesecundaria; atiendan a población en situación de vulnerabilidad o en contextos de riesgo social; presenten bajos niveles de logro educativo o altos índices de deserción escolar; estén ubicadas en municipios y localidades en los que opere el Programa Nacional para la Prevención Social de la Violencia y la Delincuencia y en la Cruzada Contra el Hambre (R0, 2015), y la segunda es que escuelas ha solicitado su ingreso al programa, si cumplen con al menos de uno de los cuatro criterios ya dichos, se les incorpora, lo que también depende de la disponibilidad presupuestaria.

Aún con estos datos hay algunas quejas del proceso de ingreso, la más consistente es la resistencia a cumplir con la extensión del horario, si bien no es significativa, ocurre donde hay una alta rotación de docentes, cuando dicho fenómeno ocurre consistentemente en una escuela de los 
conoce como "de paso", indica que los docentes están solo temporalmente mientras logran ser adscritos a una institución afín a sus intereses.

En cuanto al conocimiento y dominio del programa hay versiones disímbolas, pues mientras existen evidencias de cursos de capacitación y entrega constante de materiales educativos, en la realidad eso no se refleja. Aquí es muy probable que esté ocurriendo que la cultura magisterial, entendida como un proceso vertical y autoritario, choca con los principios de autonomía y relativa independencia que tienen los directores y docentes para utilizar los recurso y materiales educativos, así como para concretar su gestión escolar o pedagógica ya sea en la escuela o en el aula. La situación más sintomática es problema al momento de incluir la Línea de Trabajo Educativo en la planeación didáctica.

En lo que respecta al logro educativo, tomando como referentes los resultados de OCI en periodo de 2013-2017. En conjunto las dos primeras escuelas que iniciaron en el programa, en el periodo de 2013-2014, establecieron una línea base de $35 \%$ de aciertos. Para los años posteriores se toman en cuenta las 39 instituciones que se completaron en el año de 2016-2017.

Tabla 1

Resultados de OCI de las escuelas del PETC periodo 2013-2017.

\begin{tabular}{cc}
\hline Ciclo Escolar & \% obtenido en OCI \\
\hline $2013-2014$ & 35.02 \\
$2014-2015$ & 34.29 \\
$2015-2016$ & 35.07 \\
$2016-2017$ & 37.13 \\
\hline
\end{tabular}

Fuente: Elaboración propia con datos del SEER. Departamento de Evaluación. (2014, 2015, 2016, 2017). Concentrado OCI.

Como se puede observar al año siguiente de que se estableció la línea base hubo un ligero descenso de $.73 \%$, para que en los dos años siguientes se manifestara un repunte que llegó a ser de 2.11 \% con respecto a la línea base. Como la mayoría de las escuelas ingresaron en el ciclo 2014-2015, fue con la evaluación de OCI 2015-2016 y 2016-2017, que se pudo hacer un balance más general para identificar las que avanzaban, las que retrocedían y aquellas que se mantenían en sus rangos. 
Tabla 2

Escuelas que avanzan y retroceden del PETC en OCI.

\begin{tabular}{ccc} 
Resultado & OCI 2016 & OCI 2017 \\
\hline Avanzan & 17 & 29 \\
Sin cambio & 5 & 1 \\
Sin dato & 1 & 0 \\
Retroceden & 14 & 9 \\
Totales & 37 & 39
\end{tabular}

Fuente: Elaboración propia con datos del SEER. Departamento de Evaluación. (2014, 2015, 2016, 2017). Concentrado OCI.

Los resultados de OCI 2016 dicen que; 17 escuelas incrementaron el logro educativo (LE) con respecto a la línea base. En 14 él LE descendió. En cinco el LE se mantuvo, no subió, pero tampoco bajó. una escuela no tuvo alumnos para registrar los datos, y en una no se tuvieron resultados pues era su primer año en el programa. Para el año siguiente los resultados habían mejorado notablemente, pues 29 avanzaron 1 no tuvo cambios, y solamente 9 habían retrocedido.

Además de los resultados obtenidos en la OC de tres años consecutivos (periodo 2013-2017), durante mayo 2018 se llevó a cabo una jornada de visitas para observar y acompañar a las escuelas inscritas en el PETC. Para tal efecto, se emprendieron recorridos de trabajo de campo en las instituciones escolares pertenecientes a la zona 13 ubicada en la Huasteca Potosina (Tamazunchale, S.L.P.), la cual consta de trece escuelas de las 39 que conforman el total de las escuelas del SEER, es decir el 33.33\%.

Para complejizar el análisis y ampliar la comprensión del fenómeno educativo, en dicho trabajo se realizaron observaciones de la práctica docente dentro del aula, se aplicaron cuestionarios a estudiantes y padres de familia. Asimismo, se observaron las condiciones materiales de las escuelas para dar cuenta de la aplicación física de los recursos financieros provenientes del programa en cuestión (PETC). Esto último quedó documentado en registros fotográficos. La primera etapa de esta jornada de visitas duró dos días. Un tercer día se destinó a observar las reuniones de Consejo Técnico y Jornada Ampliada; al final, en plenaria, se informó a la comunidad educativa de la zona 13 (directores y docentes) los primeros resultados obtenidos los dos días anteriores.

Tomando como base que la OC se aplica a los alumnos de $6^{\circ}$ año, para las observaciones en el aula, se seleccionaron los grupos de $5^{\circ}$. Para tal motivo, se decidió registrar fortalezas y áreas de oportunidad en la práctica

Escuelas de tiempo completo y logro educativo: experiencias de las escuelas... 
docente, así como observar dinámicas, comportamientos y niveles de aprendizaje manifiestos por los alumnos de dicho grado; todo con la finalidad de ir apuntalando el proceso de mejora educativa del próximo ciclo escolar (2018-2019).

La observación fue guiada por un cuestionario construido para tal efecto: Instrumento sobre la Valoración de la Implementación del PETC (IVIP). El cuestionario valora tres dimensiones: jornada ampliada, implementación de las líneas de trabajo educativo y apoyo al aprendizaje de los niños. Adicional a esto, se aplicaron cuestionarios especiales diseñados para padres de familia y alumnos. Ambos grupos los integraron ocho sujetos previamente seleccionados por la dirección de cada escuela; para el caso de los alumnos, se pidió provinieran de los grados 4to, 5to y 6to. En el grupo de padres de familia, las preguntas giraron en torno al servicio de alimentación. En el grupo de alumnos, además del servicio alimenticio otorgado por la institución, las preguntas se enfocaron en conocer sus percepciones de satisfacción con la escuela y los profesores.

A continuación, se presentan algunos de los principales hallazgos centrados en los dos ejes que interesan analizar por constituir el soporte pedagógico para lograr la mejora educativa. Dichos ejes se relacionan con el uso eficiente de la jornada escolar e implementación de las líneas de trabajo educativo propuestas por el PETC.

\section{Uso eficaz de la jornada escolar}

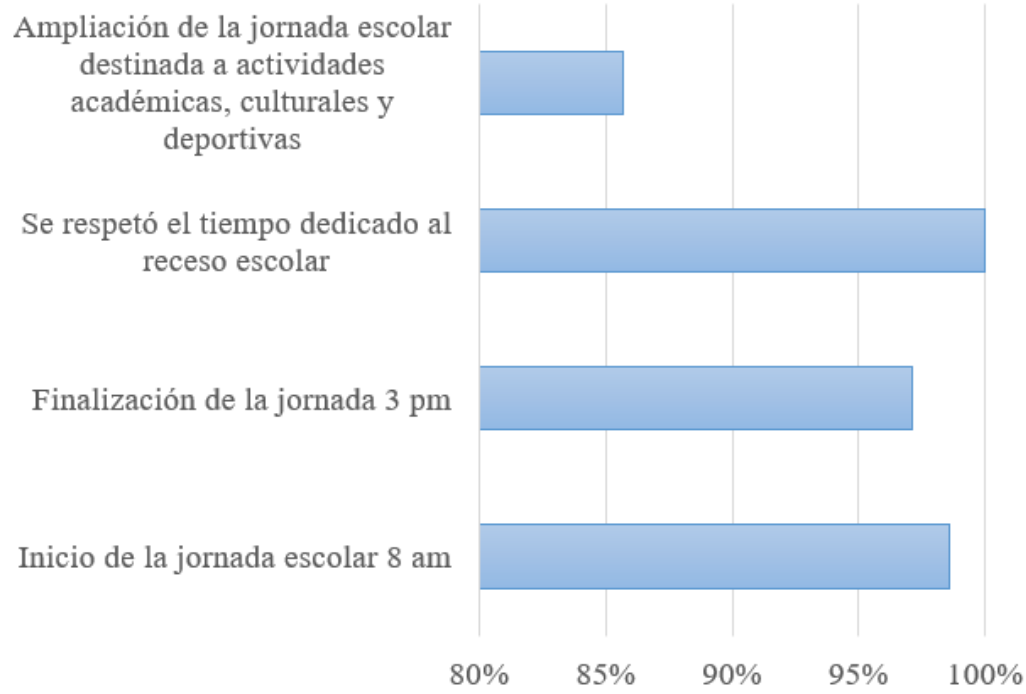

Figura 1. Eje 1. Uso eficaz de la jornada escolar

Espinosa Hernández, A.R.; y Álvarez Cooper, O.L. 
Como se puede ver en la figura 1, inicio y conclusión de la jornada escolar son, en términos generales, puntuales; así también el receso escolar es respetado en su totalidad en las escuelas visitadas. De acuerdo con los datos obtenidos, se requiere fortalecer el tiempo utilizado en actividades académicas, deportivas y culturales para aprovechar de manera más eficiente la jornada escolar ampliada. La figura 2 confirma la afirmación anterior, muestra que $79 \%$ de las instituciones educativas observadas dedican entre $75 \%$ y $100 \%$ de su jornada para realizar actividades pedagógicas y curriculares; $21 \%$ de las escuelas ocupan entre $50 \%$ y $74 \%$ de su tiempo en ese tipo de actividades.

\section{Uso eficaz de la jornada escolar en actividades pedagógicas y curriculares}

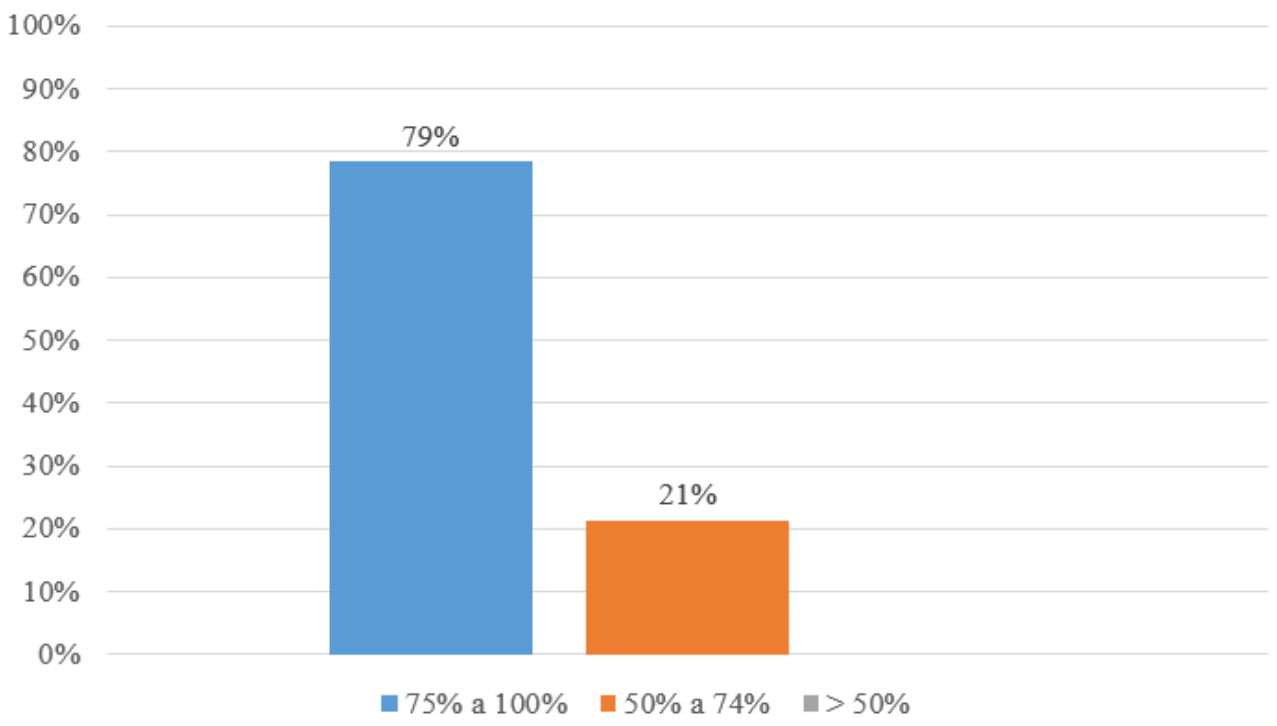

Figura 2. Eje 1. Uso eficaz de la jornada escolar en actividades pedagógicas y curriculares.

En lo referente a la implementación de líneas de trabajo propuestas por el PETC, las mayores fortalezas identificadas fueron en los rubros de planeación didáctica del día y elaboración de productos por parte de los alumnos derivados de las actividades orientadas por el profesor; de igual manera, sobresale la utilización de libros de texto de la SEP como principales auxiliares del proceso enseñanza-aprendizaje dentro del aula. Las áreas que precisan mayor atención por parte del cuerpo académico de cada escuela tienen que ver con reforzar la articulación de la estrategia educativa que ofrece el PETC con la planeación didáctica y praxis docente.

Escuelas de tiempo completo y logro educativo: experiencias de las escuelas... 
RECIE. Revista Electrónica Científica de Investigación Educativa Vol. 4, núm. 2, enero-diciembre 2019, pp. 943-954.

Esto con la finalidad de integrar adecuadamente contenidos curriculares, líneas de trabajo, ficheros didácticos y actividades realizadas con los alumnos para potenciar su efecto y así, crear una sinergia de elementos humanos y pedagógicos que se traduzcan en una mejora educativa tangible.

\section{Implementación de líneas educativas PETC}

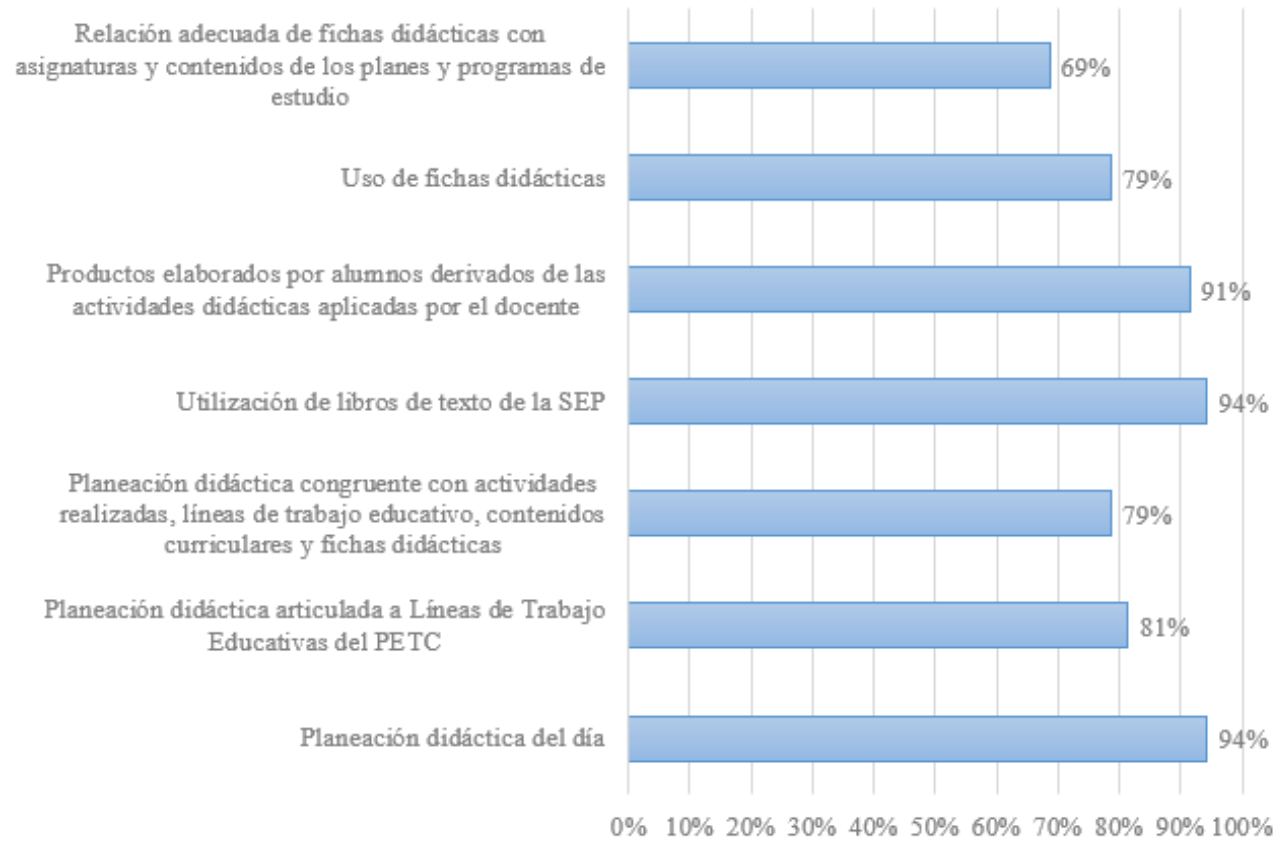

Figura 3. Eje 2. Implementación de líneas de trabajo educativo PETC.

\section{Conclusiones}

El Estado mexicano ha manifestado una creciente preocupación por revertir los malos resultados del sistema educativo nacional, a partir de la implementación de políticas educativas focalizadas para atender a población en condiciones de vulnerabilidad social. Para los cual se han tomado en cuenta las experiencias internacionales.

La implementación de programas que tienen como finalidad mejorar el logro educativo en condiciones de vulnerabilidad social, están condicionados por el conocimiento y dominio de quienes son responsables de operarlos, en parte influye la cultura institucional de las estructuras burocráticas. 
Los procesos de gestión educativa tienen que ser más articulados para garantizar que estrategias como el PETC funcionen y los resultados sean mejores, implicando la profesionalización de los agentes educativos.

Partiendo de los datos de OCI se puede afirmas que el PETC en el contexto de estas 39 escuelas revisadas, ha tenido ciertos logros, pues tres de cada 10 escuelas han mejorado y solo cuatro están en una situación crítica. Sin duda es necesario seguir haciendo esfuerzos para que nuestras escuelas sean lugares donde a los alumnos se le dé una educación que responda a sus expectativas y los prepare para participar exitosamente en la sociedad, y no condenarlos al círculo eterno de la reproducción de la pobreza.

\section{Referencias}

ACUERDO número 18/12/15 por el que se emiten las Reglas de Operación del Programa Escuelas de Tiempo Completo para el ejercicio fiscal 2016, Diario Oficial de la Federación. Domingo 27 de diciembre de 2015.

Gobierno de la República. (s. f.). Plan Nacional de Desarrollo 2013-2018.

Gómez, M. y Alemán, L. (2014). PROYECTO PETC: Estudio de casos de éxito sobre la gestión y el liderazgo escolar en las escuelas de tiempo completo. Monterrey, N.L: Instituto Tecnológico y de Estudios Superiores de Monterrey.

Ley General de Desarrollo Social. Diario Oficial de la Federación. 1 de junio de 2016.

López, M. (2012). El aporte pedagógico del PETC a la calidad en el proceso enseñanza-aprendizaje en una escuela primaria en Hidalgo. Tiempo de Educar, 13 (25), 113-136.

Martinic, S. (2015). El tiempo y el aprendizaje escolar la experiencia de la extensión de la jornada escolar en Chile. Revista Brasileira de Educação, 20 (61), 479-499.

Pozner, P. (1997). "La gestión escolar". En El directivo como gestor del aprendizaje (pp., 69-91). Buenos Aires: Editorial Aique.

Programa Sectorial de Educación 2013 - 2018. Diario Oficial de la Federación. Viernes 13 de diciembre de 2013.

Ramírez, F. (2014). Sobre la extensión de la jornada escolar en Uruguay: ocho supuestos de la política educativa en debate. Revista Latinoamericana de Estudios Educativos (México), XLIV (2), 13-38.

SEER. Departamento de Evaluación. (2014, 2015, 2016, 2017). OCI Concentrados. Autor.

Escuelas de tiempo completo y logro educativo: experiencias de las escuelas... 
SEP. (2009). Buenas prácticas educativas en la jornada escolar ampliada. México, Autor.

SEP. (s. f.). Lineamientos para la organización y el funcionamiento de las Escuelas de Tiempo Completo Educación Primaria. Autor.

Tenti, E. (2010). Estado del arte: Escolaridad primaria y jornada escolar en el contexto internacional. Estudio de casos en Europa y América Latina. Buenos Aires SEP: IIPE. UNESCO Sede Regional Buenos Aires.

Veleda, C. (2013). Nuevos tiempos para la educación primaria: lecciones sobre la extensión de la jornada escolar. Buenos Aires: Fundación CIPPEC; Unicef Argentina. 